Pre-print copy of paper published: Castellazzi MS, Wood GA, Burgess PJ, Morris J, Conrad KF \& Perry JN (2008). A systematic representation of crop rotations. Agricultural Systems 97: 26-33.

\title{
A systematic representation of crop rotations
}

\author{
M.S. Castellazzi ${ }^{1}$, G.A. Wood ${ }^{2}$, P.J. Burgess ${ }^{2}$, J. Morris ${ }^{2}$, K.F. Conrad ${ }^{1} \&$ J.N. Perry ${ }^{1}$ \\ ${ }^{1}$ PIE Division, Rothamsted Research, West Common, Harpenden, Hertfordshire, AL5 2JQ, UK, and \\ ${ }^{2}$ Natural Resources Department, Cranfield University, Cranfield, Bedfordshire, MK43 OAL, UK
}

\begin{abstract}
Crop rotations are allocations by growers of crop types to specific fields through time. This paper aims at presenting i) a systematic and rigorous mathematical representation of crops rotations; and ii) a concise mathematical framework to model crop rotations, which is useable on landscape scale modelling of agronomical practices. Rotations can be defined as temporal arrangements of crops and can be classified systematically according to their internal variability and cyclical pattern. Crop sequences in a rotation can be quantified as a transition matrix, with the Markovian property that the allocation in a given year depends on the crop allocated in the previous year. Such transition matrices can represent stochastic processes and thus facilitate modelling uncertainty in rotations, and forecasting of the long-term proportions of each crop in a rotation, such as changes in climate or economics. The matrices also permit modelling transitions between rotations due to external variables. Computer software was developed that incorporates these techniques and was used to simulate landscapescale agronomic processes over decadal periods.
\end{abstract}

\section{Keywords}

crop rotation; transition matrix; stochastic modelling; crop temporal arrangement; long-term crop proportions; landscape simulation

\section{Introduction}

Crop rotation is one of the oldest and most fundamental agronomical practices (Lawes et al., 1895), and is thought to have been critical in sustaining the industrial revolution in Britain by increasing crop production (Brunt, 1999).

A rotation is "the sequence of crops grown in succession on a particular field" (Wibberley, 1996). The final choice of sequence is primarily a management decision based on a desire to optimise, for example, financial, agricultural or environmental objectives. A key financial objective might be profit maximisation, an agricultural objective might be yield maximisation from a particular mix of crops, and an environmental objective might be minimised pesticide use. Optimisation of all objectives simultaneously might not be possible, and usually the solution found is subject to various constraints, e.g. pareto optimality (Matthews et al., 2006), and constraint programming (Tarim et al., 2006). In practice, the possible sequences in a particular location may also be constrained by government regulation, agro-ecological 
conditions (e.g. the climate, topography and soil type), the presence of disease, weeds and pests, and the crop varieties and technology available.

An critical step in allocating crops in most rotations is identifying the crop most favoured by the grower. This may be the most profitable crop in a commercial system or a staple food crop in a subsistence system. In parts of the UK, this primary crop has changed from spring barley in the 1960s, through winter barley in the 1970s, to winter wheat since the 1980s (Wibberley, 1996). Growers will typically aim to grow as much of the preferred crop as possible, on a cyclical basis.

From the literature, it can be argued that there are four major types of constraint or "rules" determining crop rotations.

The first rule is a minimum return period between the same crop, or in some cases, the maximum period of growing the same crop. Such constraints typically arise because of the need to break the cycle of the build-up of nematodes or other soil pests (Jones and Perry, 1978). For example, between the 1930s and 1980s, British sugar factories required a minimum return of four years for sugar beet to minimise the effect of beet cyst nematode (Globodera schachtii; Wibberley, 1996). In 2006, Assured Produce (scheme of standards for safe food of good quality) recommended a minimum return period of at least four years, and ideally, six years between onion crops in the UK (Assured Produce, 2007). Whereas some farmers will grow a second wheat crop directly after a first wheat crop in the UK, it is less common to grow a third wheat because of the build-up of soil fungi such as take-all (Gaeumannomyces graminis).

The second rule relates to the benefits or risks of succession associated with one crop being grown immediately after another. The benefits could arise from increased nitrogen supply, soil organic matter or water availability, improvements in soil structure, and decreased pests, diseases or weed competition (Berzsenyi et al., 2000). For example, in the Netherlands, a leguminous crop was more likely to precede organic winter wheat than in conventional arable systems (Tamis and van den Brink, 1999). Conversely, risks might arise from increased weed problems. For example, in the UK barley is more contaminated by volunteer cereal weeds than wheat (Wray, 1993) and such volunteers can be a particular problem if cereals are followed by autumn-sown vegetable crops (Bond et al., 2006).

The third rule relates to within-year cycles. The timing of sowing and harvest are dependent on interactions between the crop and the climate, and one crop usually must be harvested before the next crop can be sown. For example, in the UK, there is a constraint against planting an autumn-sown cereal crop in the same year after a spring-sown sugar beet crop, because sugar beet harvesting usually occurs between October and January. Obviously, inter-annual variations in climate can modify the constraints operating in a given season, and therefore growers require flexibility within crop rotations to overcome such problems. Late harvesting due to low temperature or high autumn rainfall can restrict autumn cultivations to such an extent that the following crops will perforce be spring- rather than autumn-sown. Indeed, spring-sown oilseed rape is four times more prevalent in arable rotations in Scotland than in England for this reason (Champion et al., 2003).

The fourth rule concerns the overall proportions of the crops grown on a field or group of fields (Castellazzi et al., 2007). Growers typically have only a limited amount of dedicated machinery and labour. They will therefore often seek to balance the distribution of work through the cropping season by growing a range of crops. In this way, the grower can also spread the risk of total crop failure and economic loss (Lockie et al., 1995). Alternatively, within the UK there is a recent trend to grow the 
same crop on the majority of fields in a single season, to attempt to benefit from economies of scale (DEFRA, 2007). Since 1992, another feature of crop rotations in the European Union has been the obligation on growers to "set-aside" a particular proportion $(5-10 \%)$ of the total area on which they had previously grown arable crops, in order to limit crop overproduction.

Several authors have modelled mechanistically the development of rotations (Bachinger and Zander, 2006; Dogliotti et al., 2003; Maxime et al., 1996; Rounsevell et al., 2003; Stöckle et al., 2003). Others have predicted actual rotations from largescale spatial or temporal data concerning the proportions of crops grown in regions, as in the studies by Klöcking et al. (2003) and Mignolet et al (2004). Klein Haneveld and Stegeman (2005) referred explicitly to some of the agronomic rules discussed above, through which crop rotations may be developed. They used the mathematical optimisation technique of linear programming to derive rotations to assist in modelling agricultural production planning. Detlefsen (2004) presented a model which integrated elements of the optimisation and prediction approaches, by using network modelling. The above mentioned modelling approaches seek to explain, optimise or even predict rotations. However none was found to provide a relevant and simple mathematical framework to represent crop rotations in a stochastic and empirical way. Such a mathematical representation would facilitate the explicit integration of crop rotations within models considering crops within agricultural landscapes through time (Brown, 2000). Such models might aim at simulating soil erosion, diffuse pollutions, plants or animal interactions (i.e. within landscape ecology, Ben Wu and Smeins, 2000), or even coexistence scenarios of GM crops (i.e. models of gene contamination from GM to conventional varieties of a crop, Castellazzi et al., 2007) for which the temporal and spatial allocation of the crops in fields influences the simulation outputs.

Our objective is to present a systematic representation of crop rotations, in terms of well-known mathematical and rigorous statistical concepts. We describe empirically determined crop rotations in a concise mathematical framework, which is amenable to large, landscape scale modelling of agronomic practices. We show how a rotation may be represented mathematically as a transition matrix and how this allows the estimation of the long term overall proportion of each crop grown. We also show how hierarchies of transition matrices may be used to represent the temporal evolution of one rotation to another, as might happen in response to crop markets or climate change.

\section{Classification of crop rotations}

The classification of crop rotations is exemplified (Figure 1(b)) by a typical arable five-year rotation for medium to heavy soils in the East Anglian region of the south-east of England (Clarke et al., 2000), described by Jim Orson (pers. comm.). Simpler (Figure 1(a)) and more complex (Figure 1(c,d)) variations on this rotation are given to illustrate further examples of terminology.

The first type of rotation (Figure 1a) is classified as 'fixed'. Each crop follows a pre-defined order with no possibility of deviation (see, for example, Colbach et al., 2005). Note that the two occurrences of wheat are identified differently; thus Wheat 1 occurs after fallow, while Wheat 2 occurs after oilseed rape (OSR). The rotation in Figure $1 a$ can also be classified as 'cyclical' in that it repeats itself. The "return period' or 'rotation length' of the rotation is also "fixed", in this case at five years.

The second rotation (Figure $1 b$ ) is classified as 'flexible' and may be represented by a "multi-pathway" network. Here, for at least one crop within the 
rotation, the grower makes a choice of which one of several crops will follow it. For example, while sugar beet would be followed optimally by late-drilled winter wheat, on clay soils, late autumn rainfall may delay sowing to the extent that the grower is forced to substitute spring-sown barley. In extreme cases of excessive winter and spring rainfall, the land may need to be left fallow for a year. Hence the choice of crops for this second year of the rotation is flexible. As in the example in Figure 1a, the rotation in Figure $1 b$ is cyclical with a fixed rotation length; each of the six potential paths is of five years duration.

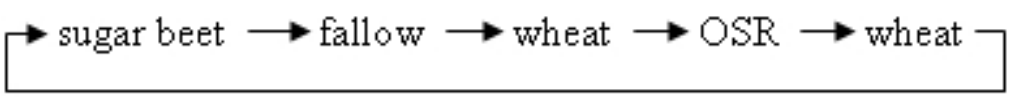

a). Fixed rotation, cyclical, fixed rotation length

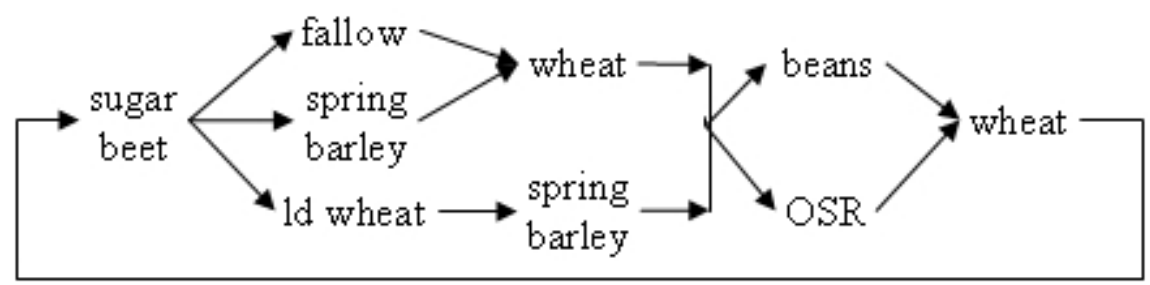

b). Flexible rotation, cyclical, fixed rotation length

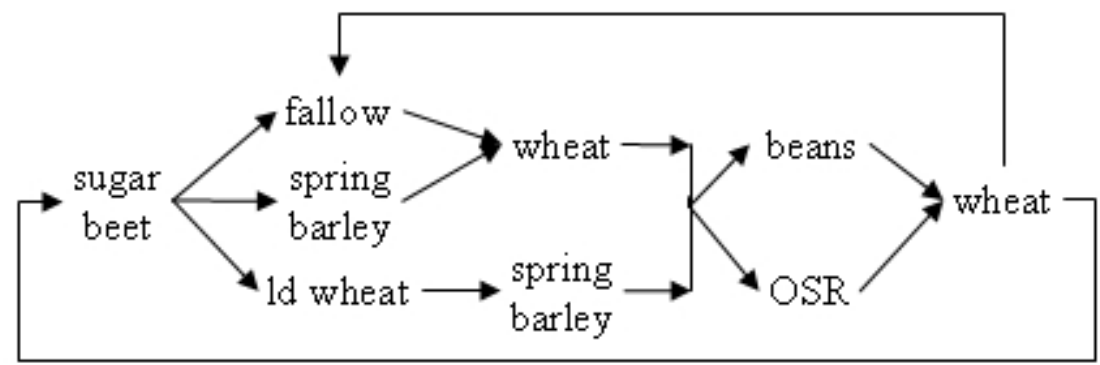

c). Flexible rotation, cyclical, variable rotation length rotation length

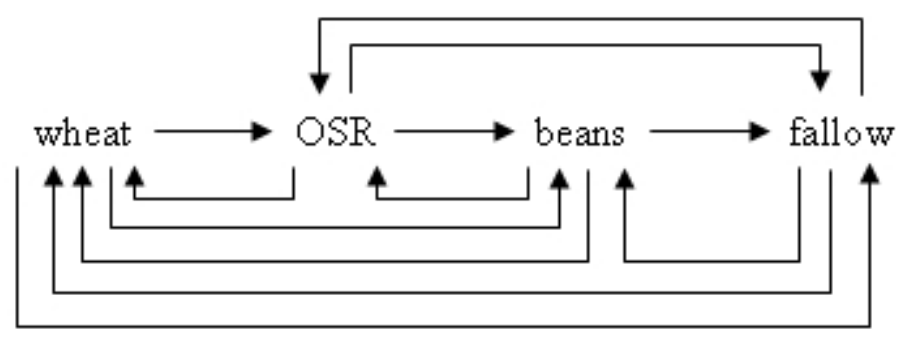

d). Flexible rotation, non-cyclical, variable

Figure 1. Examples of classified rotations a). The rotation is 'fixed'. The two occurrences of wheat are defined differently according to when they occur, as 'wheat 1' or 'wheat 2'. The rotation is 'cyclical' with a 'fixed rotation length' of five years. OSR represents oilseed rape. $b$. This more typical rotation (Orson, pers. comm.) is 'flexible' (i.e. multi-pathways). The two occurrences of spring barley are labelled accordingly. It is 'cyclical', again with a length of five years. Ld indicates late-drilled. c. This rotation is again flexible (i.e. multi-pathway), and cyclical but the rotation length is not fixed. $d$. This rotation is less-structured with great flexibility (multi-pathway), and cyclical but with a highly variable rotation length. 
In the long-term for multiple pathways rotations, the pattern of succeeding crops may be viewed as a series of chance outcomes, driven by unpredictable events such as climate or agricultural market forces that can be modelled by a stochastic process. Although a grower makes deliberate decisions based on current knowledge, simulation models usefully can represent future patterns stochastically, with probabilities derived from previous data if available.

The rotation shown in Figure $1 c$ is again flexible (i.e. multi-pathway), and cyclical, but in this example the rotation length is "variable". For example, the return period to the wheat 2 crop may be either four or five years.

The rotation in Figure $1 d$ is less structured. There is a great flexibility and the only constraint on succession is that a crop type cannot follow itself. The rotation remains cyclical but is of a highly variable rotation length. The number of possible pathways increases exponentially with years.

\section{A mathematical representation of crop rotations}

It is possible to represent most crop rotations as a set of rules in which the allocation of a crop in a current year is determined solely by the previous year's crop. As long as there is no need to consider any crop that was grown more than one year prior to the current crop, a rotation may be regarded as a Markov chain, represented by a stochastic matrix (Cox and Miller, 1965). It is therefore possible to represent the rotation as a square transition matrix, here denoted as $\mathbf{T}$. The matrix $\mathbf{T}$ has as many rows and columns as there are distinct crops, say $c$. Where a specific crop occurs more than once in a rotation these occurrences must be represented by distinct rows and columns. The element in row $i$ and column $j, t_{\mathrm{ij}}$, represents the probability under the rotation that, given that crop $i$ was grown in the previous year, there will be a transition to crop $j$ in the current year. Note that the sum of each row must be unity, so $\Sigma_{\mathrm{j}} t_{\mathrm{ij}}=1, j=1, \ldots, c$.

As an example, the rotation in Figure $1 a$ is represented uniquely by the transition matrix in Table 1a. Note how, the two wheats are distinguished as 'wheat 1' and 'wheat 2' (Figure 1). Since the rotation is fixed, the only entries possible are ' 1 ', which denotes a transition must take place, or ' 0 ' denoting it cannot. In Table 1(b), the flexible rotation given in Figure 1(b) has non-zero and non-unity values in the rows that represent a transition from a crop in the previous year that has more than one possibility of a succeeding crop in the current year (i.e. has more than one branch in Figure 1(b)), such as sugar beet. The values ascribed to $t_{\mathrm{ij}}$ for the succeeding crops (here, as examples, $0.3,0.35$ and 0.35 for fallow, first spring barley and late-drilled wheat, respectively) must sum to unity. Of course, to represent the rotation in Figure $1 c$ this matrix would only require slight amendment, to replace the probability of a transition from wheat 2 to sugar beet (currently $t_{9,1}=1$ ) by some value, $t_{9,1}=a$, say, where $0<a<1$, and the probability of a transition from wheat 2 to fallow beet (currently $t_{9,2}=0$ ) by the value $t_{9,2}=1-a$. Table $1 c$ provides one example of the lessstructured rotation shown in Figure 1(d). Further common temporal restrictions may be represented by the transition matrix model.

The restriction that successive growing of the same crop type must be represented as distinct crops (e.g. wheat 1 and 2 in the examples above) facilitates the forbidding of too many repetitions of the same crop. It may be the case, for example, that wheat may not be grown on the same field for more than three successive years, in which case the transition probability from third wheat to fourth wheat would be set to zero. However, note that the modelling of a minimum return period between successive crops of the same type, as when potatoes might be grown on the same field 
only after a gap of 8 years since the previous crop, cannot be represented through the simple transition matrix model. This is due to the limitation of the Markovian property, which considers only the previous year. Only by the imposition of further conditions expressed through additional transition matrices lagged by more than one year could the minimum return period be represented. While this is certainly possible, it is beyond the scope of the discussion presented here.

At this stage, simulations indicate that the transition matrix method outlined here are sufficiently robust not to be overly sensitive to errors in estimates, so approximate values should suffice.

The transition matrix approach can be used to determine and also model the overall proportion of each of a grower's chosen crops, aggregated in space over fields, or aggregated in time over a rotation (Castellazzi et al., 2007). If the rotation is fixed, the long-term proportion of each distinct crop grown on the field is exactly equal to $1 / c$. If the rotation is flexible then the proportion must be estimated. Fortunately, expression of a flexible rotation as a transition matrix allows the long-term average proportion of each crop to be estimated over the rotation, which is addressed in the next section.

\section{Estimation of long-term proportions of crops}

The long-term proportion of each of the crops represented in a transition matrix may be estimated using well-known properties of matrices specified, for example, in sections 3.2-3.4 of Cox and Miller (1965). Suppose the distribution of crops in year $n$ is denoted by the vector $\mathbf{C}_{(\mathrm{n})}$, so that in year $n$ the proportion of crop type $j, p_{\mathrm{j}}$, is represented by the $j$ th element of $\mathbf{C}_{(\mathrm{n})}$. Following the definition of the transition matrix $\mathbf{T}$, we have $\mathbf{C}_{(2)}=\mathbf{T} * \mathbf{C}_{(1)}$, where the asterisk represents a matrix multiplication operator. More generally $\mathbf{C}_{(\mathrm{n})}=\mathbf{T} * \mathbf{C}_{(\mathrm{n}-1)}=\mathbf{T} * \mathbf{T} * \mathbf{C}_{(\mathrm{n}-2)}=\mathbf{T}^{\mathrm{n}-1} * \mathbf{C}_{(1)}$. Cox \& Miller (1965) discuss the conditions under which powers of the transition matrix converge, and then when $m$ is sufficiently large we have, approximately $\mathbf{T}^{\mathrm{m}}=$ $\mathbf{T}^{\mathrm{m}+1}=\mathbf{Q}$, say, where $\mathbf{Q}$ represents the converged transition matrix after selfmultiplication. From this it follows that the vector $\mathbf{C}_{(\mathrm{m})}$ also converges for large enough values of $m$ since $\mathbf{C}_{(\mathrm{m})}=\mathbf{Q} * \mathbf{C}_{(1)}$. Specifically, we find that the transition matrix $\mathbf{T}_{(\mathrm{n})}$ converges to $\mathbf{Q}$ as $n$ increases, so that the $c$ row elements in any particular column become ever more similar. The average of the $j$ th column, $p_{\mathrm{j}}=\Sigma_{\mathrm{i}} t_{\mathrm{ij}(\mathrm{n})} / c$, then represents the long-term average proportion, $p_{\mathrm{j}}$, of the crop type represented by column $j$. In practice, care must be taken to ensure that algorithms implementing this technique do not suffer from round-off error. For general transition matrices Cox \& Miller (1965) explain how it may be possible to use the eigenvalues and eigenvectors of the matrix $\mathbf{T}$ to aid computation, but caution is required if the rotation has a fixed return period. Then the matrix $\mathbf{T}_{(\mathrm{n})}$ will converge to a stable set of $c$ matrices rather than a single matrix, where $c$ is equal to the number of distinct crops and, therefore, to the return period of the rotation. If this is the case, then the method is identical but the proportions are computed by averaging the columns over $c$ successive matrices: $\mathbf{T}_{(\mathrm{n})}$ to $\mathbf{T}_{(\mathrm{n}+\mathrm{c})}$.

As an example, consider the method applied to rotation (b) in Figure $1 b$ and represented by the stochastic transition matrix in Table $1 b$. The stable set of nine converged matrices gave the following long-term average proportions, $p_{\mathrm{j}}$. For sugar beet $(j=1): p_{1}=0.2$, fallow: $p_{2}=0.06$, spring barley $1: p_{3}=0.07$, late-drilled wheat: $p_{4}=0.07$, wheat $1: p_{5}=0.13$, spring barley $2: p_{6}=0.07$, beans: $p_{7}=0.1$, oilseed rape: $p_{8}=0.1$, wheat $2: p_{9}=0.2$. Note that in this fairly simple example sugar beet always 
occurs in the first year of the rotation and, since the rotation length is five years, it occurs once every five years, i.e. with a proportion of 0.2 . Furthermore, the proportion of the crops featuring in the following years are in direct proportion to their probability of succeeding sugar beet, i.e. to the probability of travelling down their particular branch in the network of Figure $1 b$. In this way the results obtained may be seen to be intuitive. However, note also that it may be desirable to know the proportions of crops per se, with no distinction of their order in the rotation. In this case, wheat has an overall proportion of $p_{4}+p_{5}+p_{9}=0.07+0.13+0.20=0.40$, and spring barley an overall proportion of $p_{3}+p_{6}=0.07+0.07=0.14$. A further example is given by the unstructured rotation (d) shown in Figure $1 d$ and represented by the stochastic transition matrix in Table $1 c$. The result is a rapid convergence to a single matrix with, in this example, the long-term average proportions for wheat as $p_{1}=0.243$; fallow, $p_{2}=0.210$; oilseed rape, $p_{3}=0.257$; and beans, $p_{4}=0.290$.

Table 1. Examples of transition matrices

a) A transition matrix, $\mathbf{T}$, can uniquely represent the rotation in Figure 1a; for any fixed rotation such as this the matrix is populated with entries that are either zero or unity.

\begin{tabular}{lllllll}
\hline & & Current year & & & \\
& Sugar beet & Fallow & Wheat 1 & OSR & Wheat 2 \\
\hline Previous & Sugar beet & 0 & 1 & 0 & 0 & 0 \\
year & Fallow & 0 & 0 & 1 & 0 & 0 \\
& Wheat 1 & 0 & 0 & 0 & 1 & 0 \\
& Oilseed rape (OSR) & 0 & 0 & 0 & 0 & 1 \\
& Wheat 2 & 1 & 0 & 0 & 0 & 0 \\
\hline
\end{tabular}

b). One example of a stochastic matrix that represents the flexible rotation in Figure 1b; rows with entries that are not identically zero or unity represent crops in the previous year that may be followed by more than one crop in the current year.

\begin{tabular}{|c|c|c|c|c|c|c|c|c|c|c|}
\hline & & \multicolumn{9}{|c|}{ Current year } \\
\hline & & $\begin{array}{c}\text { S. } \\
\text { beet }\end{array}$ & Fallow & $\begin{array}{c}\text { S. } \\
\text { barley } 1\end{array}$ & $\begin{array}{c}\text { I.d. } \\
\text { wheat }\end{array}$ & $\begin{array}{c}\text { wheat } \\
1\end{array}$ & $\begin{array}{c}\mathrm{S} . \\
\text { barley } 2\end{array}$ & Beans & OSR & $\begin{array}{c}\text { wheat } \\
2 \\
\end{array}$ \\
\hline Previous & S. beet & 0 & 0.3 & 0.35 & 0.35 & 0 & 0 & 0 & 0 & 0 \\
\hline year & Fallow & 0 & 0 & 0 & 0 & 1 & 0 & 0 & 0 & 0 \\
\hline & S. barley 1 & 0 & 0 & 0 & 0 & 1 & 0 & 0 & 0 & 0 \\
\hline & I.d. wheat & 0 & 0 & 0 & 0 & 0 & 1 & 0 & 0 & 0 \\
\hline & wheat 1 & 0 & 0 & 0 & 0 & 0 & 0 & 0.5 & 0.5 & 0 \\
\hline & S. barley 2 & 0 & 0 & 0 & 0 & 0 & 0 & 0.5 & 0.5 & 0 \\
\hline & Beans & 0 & 0 & 0 & 0 & 0 & 0 & 0 & 0 & 1 \\
\hline & OSR & 0 & 0 & 0 & 0 & 0 & 0 & 0 & 0 & 1 \\
\hline & wheat 2 & 1 & 0 & 0 & 0 & 0 & 0 & 0 & 0 & 0 \\
\hline
\end{tabular}

c). An example of the unstructured rotation in Figure 1d; all transitions are stochastic and no rows have entries that are all identically zero or unity. Each row of a transition matrix sums to unity.

\begin{tabular}{llllll}
\hline & & \multicolumn{3}{l}{ Current year } & \\
\cline { 3 - 6 } & & Wheat & Fallow & OSR & Beans \\
\hline Previous year & Wheat & 0 & 0.3 & 0.2 & 0.5 \\
& Fallow & 0.2 & 0 & 0.3 & 0.5 \\
& Oilseed rape (OSR) & 0.5 & 0.25 & 0 & 0.25 \\
& Beans & 0.25 & 0.25 & 0.5 & 0 \\
\hline
\end{tabular}

Knowledge of the long-term average for each rotation can help a grower to achieve a desired balance of crop proportions. For example, suppose a grower has 10 equally-sized fields under rotation (b), in some of which it is proposed to switch to rotation (d). Furthermore, assume that the grower should achieve an average proportion of oilseed rape of at least 0.2 . Clearly, since $p_{8}=0.1$ for rotation (b), the 
expected proportion of oilseed rape if $f$ fields are put under rotation (d) varies from the current value (with $f=0$ ) of 0.1 to a value of 0.257 (with $f=10$, from the value of $p_{3}$ for rotation $\left.(d)\right)$. So, in general, the expected proportion of oilseed rape is: $[0.1(10-f)+0.257 f] / 10$. Then it can be seen that the grower must put at least $f=7$ fields into rotation (d) to achieve the desired minimum proportion. Note, however, that an increase in one crop proportion always will come at the cost of a decrease in another, as is the case here for wheat, which for $f=7$ would decline in proportion from 0.40 to about 0.29 . To optimise over several crop proportions at once requires further information concerning costs and benefits and is a complex linear programming problem which may be solvable, but is outside the scope of this paper.

\section{Transitions between rotations}

In this section a further extension to the representation of rotations as transition matrices is introduced. This facilitates the modelling of a process where a grower might wish to change between several rotations, for example to maximise profit or to minimise pest and disease pressure. The ideas are best illustrated with a simple example concerning two fixed rotations (Figure 2). The first rotation, R1, is of length three years, with wheat followed by oilseed rape followed by beans. The second, R2, is of length two years, with wheat followed by oilseed rape. Clearly, the proportion of each crop in R1 is one third and of each crop in R2 is 0.5 , and we have seen above how the rotations may be represented by a $3 \times 3$ and a $2 \times 2$ transition matrix, respectively. Currently in the UK, beans represent a much less profitable crop than either wheat or oilseed rape. However, the three-crop rotation with beans might have the advantage of a lower incidence of pests and diseases. A grower might then have reasons to switch between these rotations, especially given the need to respond quickly to market forces. This switching may itself be represented by a $2 \times 2$ transition matrix, an example of which is shown in Table $2 a$. Here, the probabilities of staying within the current rotations are $r$ and $s$ for rotations R1 and R2, respectively. All rows of such transition matrices sum to unity, so the probabilities of a change from $\mathrm{R} 1$ to $\mathrm{R} 2$ is $1-r$, and from $\mathrm{R} 2$ to $\mathrm{R} 1$ is $1-s$.

The system now consists of three transition matrices, which is mathematically cumbersome. Fortunately however, this may be simplified by representing the three matrices as a single transition matrix, which we denote here by $\mathbf{U}$ (Table $2 b$ ). The overall transition matrix $\mathbf{U}$ is $5 \times 5$, composed of four blocks that represent the transitions between individual crops of rotation R1 ( $3 \times 3$ top left block), R2 $(2 \times 2$ bottom right block), R1 to R2 ( $3 \times 2$ top right block) and R2 to R1 ( $2 \times 3$ bottom left block). The entries in the first two square blocks of $\mathbf{U}$ are a copy of the individual transition matrices R1 and R2, multiplied by the probability of remaining within their respective rotations. Each entry in the third and fourth rectangular blocks of $\mathbf{U}$ represents the probability of a change from a crop in one rotation to a crop in a different rotation and for simplicity it is assumed that when such an event occurs the crop in the new rotation is chosen at random, although this is not a strictly necessary condition. In this case, each entry in the third and fourth blocks of $\mathbf{U}$ is constructed by dividing the probability of changing from one rotation to the other by the number of crops in the new rotation. This process may easily be generalised to transitions between more than two rotations; the single overall matrix $\mathbf{U}$ that results will always be square with the number of rows and columns equal to the sum of the number of crops over all the individual rotations. 
a). Rotation 1 (R1)

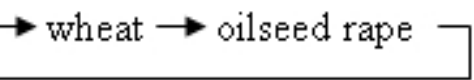

b). Rotation $2(R 2)$

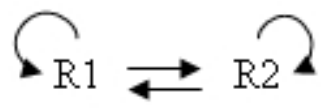

c). Transitions between rotations $R 1 \& R 2$

Figure 2. Schematic diagrams of transitions between example rotations $a$ ). Rotation 1 is a three year rotation. $b$ ). Rotation 2 is a two year rotation. $c$ ). Schema of all possible transitions between rotation 1 and 2 .

\section{Table 2. Transitions between rotations}

a) Example of a simple transition matrix representing the probabilities of transitions between two rotations, R1 and R2. The probabilities of staying within the current rotations are $r$ and $s$ for rotations R1 and R2, respectively. All rows of such transition matrices sum to unity.

\begin{tabular}{llcc}
\hline & & \multicolumn{2}{c}{ Current year } \\
& & Rotation R1 & Rotation R2 \\
\hline Previous year & Rotation R1 & $r$ & $1-r$ \\
& Rotation R2 & $1-s$ & $s$ \\
\hline
\end{tabular}

b). When the rotation R1 is a fixed three-year sequence of wheat, oilseed rape (OSR) and beans, and the rotation R2 is a fixed two-year sequence of wheat and oilseed rape, the three transition matrices representing R1, R2 and the transition between them described in $a$, may all be represented by the single $5 \times 5$ overall transition matrix, $\mathbf{U}$. The entries in the matrix $\mathbf{U}$ are formed by considering the four blocks shown, that represent the transitions between individual crops of rotation R1 ( $3 \times 3$ top left block), R2 $(2 \times 2$ bottom right block), R1 to R2 $(3 \times 2$ top right block) and R2 to R1 $(2 \times 3$ bottom left block). See text for further details.

\begin{tabular}{|c|c|c|c|c|c|c|c|}
\hline & & & \multicolumn{5}{|c|}{ Current year } \\
\hline & & & \multicolumn{3}{|c|}{ (R1) } & \multicolumn{2}{|c|}{ (R2) } \\
\hline & & & Wheat & OSR & Beans & Wheat & OSR \\
\hline \multirow[t]{5}{*}{ Previous year } & (R1) & Wheat & 0 & $r$ & 0 & $(1-r) / 2$ & $(1-r) / 2$ \\
\hline & & OSR & 0 & 0 & $r$ & $(1-r) / 2$ & $(1-r) / 2$ \\
\hline & & Beans & $r$ & 0 & 0 & $(1-r) / 2$ & $(1-r) / 2$ \\
\hline & (R2) & Wheat & $(1-s) / 3$ & $(1-s) / 3$ & $(1-s) / 3$ & 0 & $s$ \\
\hline & & OSR & $(1-s) / 3$ & $(1-s) / 3$ & $(1-s) / 3$ & $s$ & 0 \\
\hline
\end{tabular}

As an example, consider the above system, with $r=0.7$ and $s=0.9$. The greater chance of transition from R1 to R2 than vice-versa is reflected in the longterm probability of being in each rotation in a given future year, which are calculable using the methods in section 4 (i.e. matrix multiplications), and which are 0.25 for R 1 and 0.75 for R2. The long-term average crop proportions for the three crops are 0.458 for both wheat and oilseed rape (composed of $0.75 / 2+0.25 / 3$ ) and 0.083 for beans 
(composed of $0.25 / 3$ ). This strategy provides proportions of wheat and oilseed rape considerably closer to those of R2 ( 0.5 each) than to R1 ( 0.333 each).

Another example of interest is where one of $r$ or $s$ is unity, which models the situation where, once a transition to another rotation has been made, this becomes fixed, with no possibility of return. For example, consider the case of $r=0.7$, as before, but now with $s=1$, so that a transition may occur from R1 to R2 but not viceversa. Hence, in the long term, the probability of still being in rotation R1 approaches ever closer to zero (indeed, by the end of the tenth year it is less than 0.03 ).

Furthermore, since all the column elements that relate to the three crops of R1 of the matrix $\mathbf{U}^{\mathrm{n}}$ (see section 4 , above) have the form $0.7^{\mathrm{n}}$, these elements tend to zero as $n$ increases. Hence, in the long term, the proportion of crops in rotation R1 declines to zero and the only proportions relevant are those for crops in rotation R2, which are 0.5 for both wheat and oilseed rape.

A final example is presented to model the situation where a change of rotation from R1 to R2 becomes ever more likely due to some external driving trend, such as climatic change. Here, for simplicity, we again disallow a reversion to rotation R1 once a change has been made from R1 to R2, so $s=1$. But now, $r$ is a function of time. For example, consider the situation where a transition from R1 to R2 would have been unthinkable at the beginning of the present century, but the probability of which increases steadily year by year until, by 2050, it becomes inevitable. This may be modelled by the equation: $r=(2050-Y) / 50$, where $Y$ represents the current year. It is easy to substitute this variable value for $r$ into software that implements an algorithm to model such change.

\section{Discussion}

Crop rotations support sustainable crop production and soil quality over time by structuring the temporal succession of crops in fields. Rotations control the temporal patterns of crops, they are a major component of agricultural landscapes. Four groups of rotations based on the flexibility of their structure were proposed in this paper. These ranged from crop successions fixed in advance to unformed rotation which can be responsive to fluctuating environmental or market conditions. Models of agricultural landscapes incorporating spatially realistic variation in allocation of crops through time require the integration of the above mentioned rotation groups. The transition matrix technique presented in this paper provides such models with a robust and flexible tool to manage crop rotations in a mathematical framework.

The rotations are represented as probabilities of any crop grown succeeding other crops in the rotation. A major assumption of this method is that the choice of the crop to grow (year $n+1$ ) is based only upon the crop grown previously (year $n$ ). Two techniques are available to mitigate this assumption. At first, dependencies of crops over several years can be imposed with fixed crop rotations (binary probabilities, i.e. only one fixed crop rotation available), or by separating the instances of a crops (e.g. wheat crop present twice in a transition matrix to separate the contexts of its occurrences). Secondly, in a model, the use of transition matrices can be complemented with stand-alone rules restricting crop successions over any required time length. Such rules can be (i) restrictions on return period of crops, to prevent untimely growing of crops; (ii) forbidden crop sequences, to avoid unacceptable successions of crops in a field; or (iii) maximum acceptable repetitions of a crop, to prevent continuous growing of that crop.

By using the properties of the transition matrices, the long-term crop proportions of any rotation can be determined. This possibility is of particular 
importance for crop rotation modelling at the landscape scale. By knowing the longterm crop proportions of each potential rotation and the areas of each field, rotation allocation to fields can be optimised towards specific user-defined crop proportions over the whole landscape. This provides a means to control crop proportions at the landscape scale, and is essential to generate coherent crop allocation at the landscape level.

The creation of transition matrices adapted to the agricultural landscape under study requires expert knowledge on the type of crop rotation to model, and an understanding of the internal dynamics of crop successions. Such knowledge may be derived from research on farmers' decision-making of crop succession, agronomical advisers or even from statistical analyses of agricultural censuses from modelling past or present agronomical practices. For modelling crop rotations in evolving landscapes, (e.g. due to climate change or market fluctuations) the transition matrix method can be adapted to incorporate a dependency upon an external variable, as in the example relating probabilities of growing a crop to long-term temperature variation, or to allow the transition between different crop rotations. Therefore, the matrices provide the opportunity to investigate potential changes in land use due to variation in favoured crops because of changing environmental or economic conditions.

Simulation models incorporating these ideas can compute and suggest strategies relating to crop rotations and temporal patterns of crops at the landscape scale. The techniques outlined here have already been incorporated in the open-source software package, LandSFACTS

(http://www.rothamsted.bbsrc.ac.uk/pie/LandSFACTS), created to model realistic crop allocation to fields to meet user-defined constraints on crop spatio-temporal patterns at the landscape scale over multiple years. The use of the transition matrices to represent crop rotations allows the software to be useable on any agricultural landscapes, regardless of the type of crops or crop rotations in place. LandSFACTS is specifically designed to support the creation of scenarios of realistic crop arrangement at the landscape scale. Such scenarios might require a specific crop proportion over the landscape, or temporal occurrence of a crop. It is currently being used by researchers to investigate the environmental and economic impacts of coexistence measures for growing GM crops on European agricultural landscapes by the EUfunded project SIGMEA (http://sigmea.dyndns.org). More specifically ongoing work is to evaluate the effect of crop patterns derived from crop rotations on the efficacy of coexistence measures. Scenarios of crop arrangement developed for this purpose are for example focusing on (i) the relative proportions of GM and conventional crops over the years, e.g. $10 \%, 50 \%$ or $75 \%$ of oilseed rape crops as GM varieties, (ii) the spatial repartition of the rotations with GM crops within the landscape, i.e. spatially limited to an area or homogeneously widespread through the landscape, (iii) temporal and spatial separation distances between crops, such as 500m imposed between GM and conventional varieties of a crop or 3 years gap required between a growing a GM and a conventional varieties of a crop. LandSFACTS will also be useful in applications such as modelling erosion or diffuse pollution, where the realistic generated crop allocations can be used as an input to more mechanistic models.

Further work is now required, in addition to the analytical methods described by Castellazzi et al. (2007), to develop methods to estimate the probabilities of transitions from crop to crop in crop rotations. 


\section{Acknowledgements}

This work was carried out within the framework of the European Union funded SIGMEA project (http://sigmea.dyndns.org/). Rothamsted Research receives grantin-aid support from the Biotechnology and Biological Sciences Research Council of the United Kingdom.

\section{References}

Assured Produce. 2007. Crop Specific Protocol: Onions (bulb and shallots). http://www.assuredproduce.co.uk/resources/000/238/075/Onions bulb_2007.pdf [Accessed: 10/05/07]

Bachinger, J. and Zander, P. 2006. ROTOR, a tool for generating and evaluating crop rotations for organic farming systems. European Journal of Agronomy 26 (2):130143.

Ben $\mathrm{Wu}, \mathrm{X}$. and Smeins, F.E. 2000. Multiple-scale habitat modeling approach for rare plant conservation. Landscape and Urban Planning 51(1): 11-28.

Berzsenyi, Z., Gyorffy, B. and Lap, D. 2000. Effect of crop rotation and fertilisation on maize and wheat yields and yield stability in a long-term experiment. European Journal of Agronomy 13(2-3): 225-244.

Bond, W., Davies, G. and Turner, R. 2006. The biology and non-chemical control of Volunteer Cereals. Henry Doubleday Research Association, Ryton http://www.gardenorganic.org.uk/organicweeds/downloads/volunteer\%20cereals. pdf. [Accessed: 21/05/07].

Brown, D.R. 2000. A Review of Bio-Economic Models. Paper prepared for the Cornell African Food Security and Natural Resource Management (CAFSNRM) Program: 102.

Brunt, L. 1999. An arbitrage model of crop rotation in 18th Century England. University of Oxford: Oxford. Oxford University Economic and Social History Series.

Castellazzi, M.S., Perry, J.N., Colbach, N., Monod, H., Adamczyk, K., Viaud, V. and Conrad, K.F. 2007. New measures and tests of temporal and spatial pattern of crops in agricultural landscapes. Agriculture, Ecosystems and Environment 118: 339-349.

Champion, G.T., May, M.J., Bennett, S., Brooks, D.R., Clark, S.J., Danieis, R.E., Firbank, L.G., Haughton, A.J., Hawes, C., Heard, M.S., Perry, J.N., Randle, Z., Rossall, M.J., Rothery, P., Skellern, M.P., Scott, R.J., Squire, G.R. and Thomas, M.R. 2003. Crop management and agronomic context of the Farm Scale Evaluations of genetically modified herbicide-tolerant crops. Philosophical Transactions of the Royal Society of London Series B-Biological Sciences 358(1439): 1801-1818.

Clarke, J., Moss, S. and Orson, J. 2000. The future for grass weed management in the UK. Pesticide Outlook: 59-63.

Colbach, N., Fargue, A., Sausse, C., and Angevin, F. 2005. Evaluation and use of a spatio-temporal model of cropping system effects on gene escape from transgenic oilseed rape varieties - Example of the GENESYS model applied to three coexisting herbicide tolerance transgenes. European Journal of Agronomy 22(4): 417-440.

Cox, D.R. and Miller, H.D. 1965. The Theory of Stochastic Processes, Chapman and Hall, London. 
DEFRA 2007. Farming and food brief: January 2007. http://statistics.defra.gov.uk/esg/publications/Monthly\%20brief/Jan07farmfoodbri ef.pdf. [Accessed: 21/05/07].

Detlefesen, N. 2004. Crop rotation modelling. Proceedings of the EWDA-04 European workshop for decision problems in agriculture and natural resources. Silsoe Research Institute, England.

Dogliotti, S., Rossing, W.A.H. and van Ittersum, M.K. 2003. ROTAT, a tool for systematically generating crop rotations. European Journal of Agronomy 19(2): 239-250.

Jones, F.G.W. and Perry, J.N. 1978. Modeling Populations of Cyst-Nematodes (Nematoda Heteroderidae). Journal of Applied Ecology 15(2): 349-371.

Klein Haneveld, W.K. and Stegeman, A.W. 2005. Crop succession requirements in agricultural production planning. European Journal of Operational Research 166: 406-429.

Klöcking, B., Strobl, B., Knoblauch, S., Maier, U., Pfutzner, B. and Gericke, A. 2003. Development and allocation of land-use scenarios in agriculture for hydrological impact studies. Physics and Chemistry of the Earth, Parts A/B/C 28(33-36): 13111321.

Lawes, Sir Bennet, J., Gilbert and Sir Henry, J. 1895. The Rothamsted Experiments. Edinburgh, Blackwood.

Lockie, S., Mead, A., Vanclay, F. and Butler, B. 1995. Factors Encouraging the Adoption of More Sustainable Crop Rotations in South-East Australia - Profit, Sustainability, Risk and Stability. Journal of Sustainable Agriculture 6(1): 61-79.

Matthews, K.B., Buchan, K., Sibbald, A.R. and Craw, S. 2006. Combining deliberative and computer-based methods for multi-objective land-use planning. Agricultural Systems 87 (1):18-37.

Maxime, F., Nicoletti, J., Leroy, P. and Papy, F. 1996. Donner de la souplesse au choix d'assolement par des rotations-cadres. Colloque Aide à la décision et choix de stratégies dans les entreprises agricoles, Laon, Inra et Conseil régional de Picardie.

Mignolet, C., Schott, C. and Benoit, M. 2004. Spatial dynamics of agricultural practices on a basin territory: a retrospective study to implement models simulating nitrate flow. The case of the Sein basin. Agronomie 24: 219-236.

Rounsevell, M.D.A., Annetts, J.E., Audsley, E., Mayr, T. and Reginster, I. 2003. Modelling the spatial distribution of agricultural land use at the regional scale. Agriculture Ecosystems \& Environment 95(2-3): 465-479.

Stöckle, C.O., Donatelli, M. and Nelson, R. 2003. CropSyst, a cropping systems simulation model. European Journal of Agronomy 18: 289-307.

Tamis, W.L.M. and van den Brink, W.J. 1999. Conventional, integrated and organic winter wheat production in The Netherlands in the period 1993-1997. Agriculture Ecosystems \& Environment 76(1): 47-59.

Tarim, S.A., Manandhar, S. and Walsh, T. 2006. Stochastic constraint programming: A scenario-based approach. Constraints 11(1): 53-80.

Wibberley, J. 1996. A brief history of rotations, economic considerations and future directions. Aspects of Applied Biology 47: 1-10.

Wray, M.W. 1993. A survey of cereal admixture in wheat and barley grain 1988 to 1990. Aspects of Applied Biology, Cambridge, UK 35(Volunteer crops as weeds): 195-206. 